\title{
Fabrication of ZnInON/ZnO multi-quantum well solar cells
}

\author{
Koichi Matsushima ${ }^{a}$, Ryota Shimizu ${ }^{a}$, Tomoaki Ide ${ }^{a}$, Daisuke Yamashita ${ }^{a}$, Hyunwoong Seo ${ }^{a}$, Kazunori Koga ${ }^{\text {a }}$ \\ Masaharu Shiratani a, Naho Itagaki ${ }^{\text {a,b }}$ \\ a Kyushu University, Fukuoka 819-0395, Japan \\ b PRESTO, Japan Science and Technology Agency, Chiyoda, Tokyo 102-0075, Japan
}

\section{A R T I C L E I N F O}

Available online $\mathrm{xxxx}$

\section{Keywords:}

ZnInON

Oxynitride

Piezoelectric effect

Photovoltaics

Multi-quantum well

Nitrogen-mediated crystallization

Magnetron sputtering

\begin{abstract}
A B S T R A C T
We report on fabrication and photovoltaic characteristics of solar cells with $\mathrm{ZnInON/ZnO} \mathrm{multi-quantum} \mathrm{wells}$ (MQWs) in the intrinsic layer of p-i-n structure by RF magnetron sputtering. We employed two kinds of $p$ layers: one is p-GaN and the other is p-Si. Under solar simulator light, the short-circuit current $\left(J_{\mathrm{sc}}\right)$ and the open-circuit voltage $\left(V_{\text {oc }}\right.$ ) of the solar cells on p-GaN templates are $1.9 \mu \mathrm{A} / \mathrm{cm}^{2}$ and $0.16 \mathrm{~V}$, whereas $J_{\mathrm{sc}}$ and $V_{\mathrm{oc}}$ are enhanced to $2.5 \mu \mathrm{A} / \mathrm{cm}^{2}$ and $0.19 \mathrm{~V}$ under simultaneous irradiation of green laser light (532 nm) and the solar simulator light. Solar cells on p-Si substrates do not show such enhancement. A possible origin of the enhancement is a large piezoelectric field generated in strained ZnInON wells coherently grown on p-GaN template.
\end{abstract}

(C) 2015 Elsevier B.V. All rights reserved.

\section{Introduction}

Solar cells with low-cost and ultra-high conversion efficiencies, so-called "third generation solar cells", have attracted attention because of the potential to bring significant reduction in the electricity generation cost [1-5]. In single band gap devices that dominate the current photovoltaic market, choice of the material composing solar cells is a trade-off between maximizing current output with a narrow band gap material and voltage output with a wide band gap material. As a result, the maximum theoretical efficiency is limited to be $30 \%$, which is well known as the Shockley-Queisser limit [6]. One promising way to overcome this limit is employing a tandem structure with several solar cells having different band gap materials. In fact, the reported efficiency of tandem type is high over 40\%, being the highest efficiency ever reported for all types of solar cells $[7,8]$. The tandem solar cells, however, face substantive problems in the current matching between the cells that are connected in series, making further increase in the efficiency challenging. An alternative approach to obtain high efficiency is to use quantum confinement effect in multi-quantum wells (MQWs). In MQW solar cells, consisting of MQWs in the intrinsic layer of p-i-n structure, the output voltage is determined by the quasi-Fermi levels in barrier material with a wide band gap, whereas the output current is dominated by well materials with a narrow band gap. Hence, the output voltage is expected to be larger than that of the cells formed only from the material of the wells [9]. Furthermore, extra absorption of light at longer wavelengths via sub-band transition can enhance the current density

E-mail address: k.matsushima@plasma.ed.kyushu-u.ac.jp (K. Matsushima). output. These advantages of MQW solar cells make their theoretical efficiency over $50 \%$ [10-18].

In this study, we fabricated solar cells with ZnInON based MQWs, taking advantages of the MQW photovoltaic behavior mentioned above. Since the conversion efficiency is highly dependent on the band gap of well layers and barrier layers, materials with tunable band gap is required for maximizing the conversion efficiency. ZnInON semiconductor is a pseudo-binary alloy of wurtzite $\mathrm{ZnO}$ and wurtizte InN. Optical measurements revealed that ZnInON has a tunability of the band gap over the entire visible spectrum and a high optical absorption coefficient of $10^{5} \mathrm{~cm}^{-1}$ [19-21]. Therefore, this compound is a promising candidate for light absorbing MQW materials in solar cells. Compared with other tunable band gap materials such as InGaN, ZnInON have two advantages in terms of the application in MQW solar cells. First, ZnInON can be fabricated by a conventional sputtering, allowing for scaling up to larger substrates with ease. In the case of InGaN, the films are generally grown by utilizing metal-organic chemical vapor deposition method, which makes them of potentially limited use for large-scale demands because of the lack of scalability. Recently, some studies have been therefore directed towards sputtering growth of InGaN. The resultant films, however, turn out to be oxidized due to the residual gases such as oxygen and water vapor that always exists in conventional sputtering chambers [22]. In contrast, because oxygen is one of the main constituents of $\mathrm{ZnInON}$, conventional sputtering chambers can be used for ZnInON deposition. The second advantage is the large piezoelectric constants of ZnInON, which brings low recombination rate of photo-generated carries in QWs because large piezoelectric field generated in QWs separates electron and holes. This large piezoelectric constant of ZnInON owes to the large piezoelectric constant of $\mathrm{ZnO}\left(\mathrm{e}_{33}: 0.96 \mathrm{C} / \mathrm{m}^{2}\right)$, which is much larger

http://dx.doi.org/10.1016/j.tsf.2015.01.012

0040-6090/@ 2015 Elsevier B.V. All rights reserved.

Please cite this article as: K. Matsushima, et al., Fabrication of ZnInON/ZnO multi-quantum well solar cells, Thin Solid Films (2015), http:// dx.doi.org/10.1016/j.tsf.2015.01.012 
than that of GaN ( $\left.\mathrm{e}_{33}: 0.73 \mathrm{C} / \mathrm{m}^{2}\right)$. Here, we demonstrate the fabrication of ZnInON/ZnO MQWs and the solar cells with the MQWs in the intrinsic layer of p-i-n structure. The photo-electric properties of the MQWs as well as the photovoltaic properties of the solar cells are evaluated through measurements of current-voltage $(J-V)$ characteristics under photo-irradiation.

\section{Experimental details}

\subsection{Fabrication of $\mathrm{ZnInON} / \mathrm{ZnO} \mathrm{MQWs}$}

The structure of ZnInON/ZnO MQWs is shown in Fig. 1(a). Seven or 15 periods of $\mathrm{ZnInON}(6 \mathrm{~nm}) / \mathrm{ZnO}(10 \mathrm{~nm})$ MQWs were deposited on two types of templates: one is single-crystalline $\mathrm{ZnO}$ films prepared on c-plane sapphire substrates, and another is poly-crystalline $\mathrm{ZnO}$ films deposited on quartz glass substrates. Here, single-crystalline $\mathrm{ZnO}$ templates were prepared by using the nitrogen-mediated crystallization (NMC) method [23-31], which is a method for fabrication of highquality epitaxial films on large lattice-mismatched substrates. Despite the large lattice mismatch (18\%) between $\mathrm{ZnO}$ and c-plane sapphire, single-crystalline $\mathrm{ZnO}$ films that have atomically flat surface were fabricated by the NMC method. Then, $\mathrm{ZnInON}$ well layers and $\mathrm{ZnO}$ barrier layers were deposited by RF magnetron sputtering in $\mathrm{Ar}-\mathrm{O}_{2}-\mathrm{N}_{2}$ atmosphere and in $\mathrm{Ar}-\mathrm{O}_{2}$ atmosphere, respectively. The substrate temperature was room one. Finally, InGa electrodes were deposited on the bottom and the top of the $\mathrm{ZnO}$ barrier layers to measure $J-V$ characteristics.

The band gap energy was determined by Tauc plots using optical reflection and transmission data obtained with a UV-visible spectrophotometer (JASCO V-530). The band gap of ZnInON was $3.1 \mathrm{eV}$, whereas the band gap of $\mathrm{ZnO}$ was $3.4 \mathrm{eV}$. The chemical composition of films was measured with a wavelength dispersive X-ray fluorescence spectrometer (Rigaku ZSX Primus II). The chemical composition ratio of ZnInON films was $(\mathrm{ZnO})_{0.97}(\operatorname{InN})_{0.03}$.
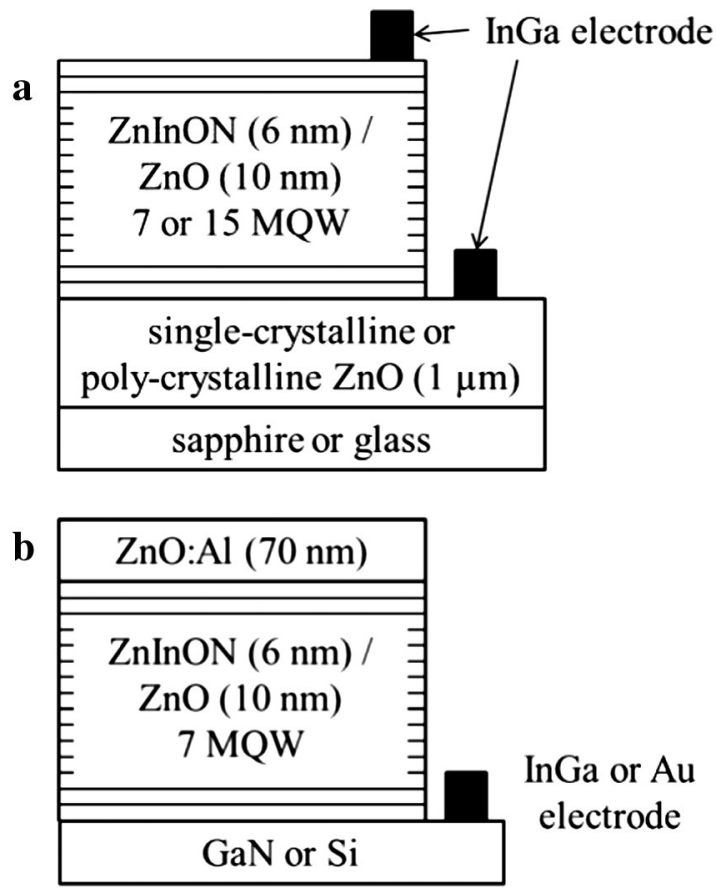

Fig. 1. Schematics of (a) ZnInON/ZnO MQWs structure, (b) solar cells with ZnInON/ZnO MQWs in the intrinsic layer of p-i-n structure.

\subsection{Fabrication of ZnInON/ZnO MQW solar cells}

The solar cell structure is shown in Fig. 1(b). The MQW solar cells were composed of n-type $\mathrm{ZnO}: \mathrm{Al}$ (AZO), 7 periods of $(\mathrm{ZnO})_{0.97}(\mathrm{InN})_{0.03}(6 \mathrm{~nm}) /$ $\mathrm{ZnO}(15 \mathrm{~nm}) \mathrm{MQWs}$, and p-type GaN template or p-type Si substrate. It is well known that $\mathrm{ZnO}$ exhibits n-type conductivity even without intentional doping, and there is difficulty in obtaining p-type because of self-compensation from native defects along with the deep acceptor levels of the dopants [32]. Therefore, p-type GaN templates, which were commercially available, were used in this study. The crystal structure as well as the band gap of $\mathrm{GaN}$ is the same as wurtzite $\mathrm{ZnO}$, and the lattice mismatch between them is significantly small of $1.9 \%$. ZnInON well layers and $\mathrm{ZnO}$ barrier layers were deposited by RF magnetron sputtering in $\mathrm{Ar}$ $\mathrm{O}_{2}-\mathrm{N}_{2}$ atmosphere and in $\mathrm{Ar}-\mathrm{O}_{2}$ atmosphere, respectively. The substrate temperature was room temperature. N-type AZOfilms of $70 \mathrm{~nm}$ in thickness were deposited on MQW layers by RF magnetron sputtering in $\mathrm{Ar}$ atmosphere. InGa electrodes were deposited on the p-GaN templates, while Au electrodes were deposited on the p-Si substrates by sputtering. No post anneal was performed.

The film thickness was measured by X-ray reflectometry. The crystallinity of ZnInON films was examined by $\mathrm{x}$-ray diffraction (XRD) analysis with a four-circle texture diffractometer (Bruker D8 Discover) using Cu $K \alpha$ radiation $(\lambda=0.15418 \mathrm{~nm}) . J-V$ characteristics were measured under AM $1.5\left(100 \mathrm{~mW} / \mathrm{cm}^{2}\right)$ and a diode-pumped-solid-state green laser light (532 nm, $2.15 \mathrm{~W} / \mathrm{cm}^{2}$ ) irradiation.

\section{Results and discussion}

\subsection{Photo-electrical properties of ZnInON/ZnO MQWs}

First, we investigated the photo response of $\mathrm{ZnInON} / \mathrm{ZnO} \mathrm{MQWs}$. Fig. 2(a) and (b) show $J-V$ characteristics of 7-period $\mathrm{ZnInON/ZnO}$ MQWs on single-crystalline $\mathrm{ZnO}$ template and on poly-crystalline $\mathrm{ZnO}$ template, respectively. The linear relationship of the $J-V$ characteristics shows that the contact between $\mathrm{ZnO}$ and InGa electrodes has ohmic character. The conductivity of the MQWs is enhanced by the irradiation of solar simulator light, indicating the absorption of the light and photoinduced generation of carriers. The photo-to-dark conductivity ratio of the MQWs on single-crystalline and on poly-crystalline $\mathrm{ZnO}$ templates are 41.8 and 1237.4, respectively, which demonstrates the high photosensitivity of the ZnInON/ZnO MQWs. We observed that the poly-crystalline $\mathrm{ZnO}$ has quite large surface roughness (root mean square roughness (RMS): $13.1 \mathrm{~nm}$ ), that is, large haze, whereas the single-crystalline $\mathrm{ZnO}$ has atomically flat surface of RMS $=0.16 \mathrm{~nm}$. Therefore, the higher current density of MQWs on poly-crystalline $\mathrm{ZnO}$ is due to the longer optical path as the result of efficient diffuse light scattering. It should be noted that there is a difference in the photo response between MQWs on single-crystalline $\mathrm{ZnO}$ and on poly-crystalline $\mathrm{ZnO}$ under irradiation of the solar simulator light together with the green laser light. The conductivity of MQWs on single-crystalline $\mathrm{ZnO}$ shows 1.8 times enhancement by superimposing the green laser light, whereas that on poly-crystalline $\mathrm{ZnO}$ does not. Since the photon energy of the laser light $(2.3 \mathrm{eV})$ is smaller than the band gap energy of the well layers $(3.1 \mathrm{eV})$, the photo-conductivity enhancement is probably due to the enhanced extraction of photogenerated carriers from the well layers.

The rather low photo-conductivity is brought about by the small thickness of MQWs and the wide band gap of the well layers. Hence, we fabricated a 15-period ZnInON/ZnO MQWs on a single-crystalline $\mathrm{ZnO}$ template, aiming to increase the photo-conductivity. The $J-V$ characteristic is shown in Fig. 2(c). As expected, the photoconductivity is increased with increasing the number of period of MQWs from 7 to 15 . The photo-to-dark conductivity ratio is high of 54.1. However, the photo-conductivity shows only 1.2 times enhancement by superimposing the green laser light. The reason of this small enhancement will be discussed in the next section. 

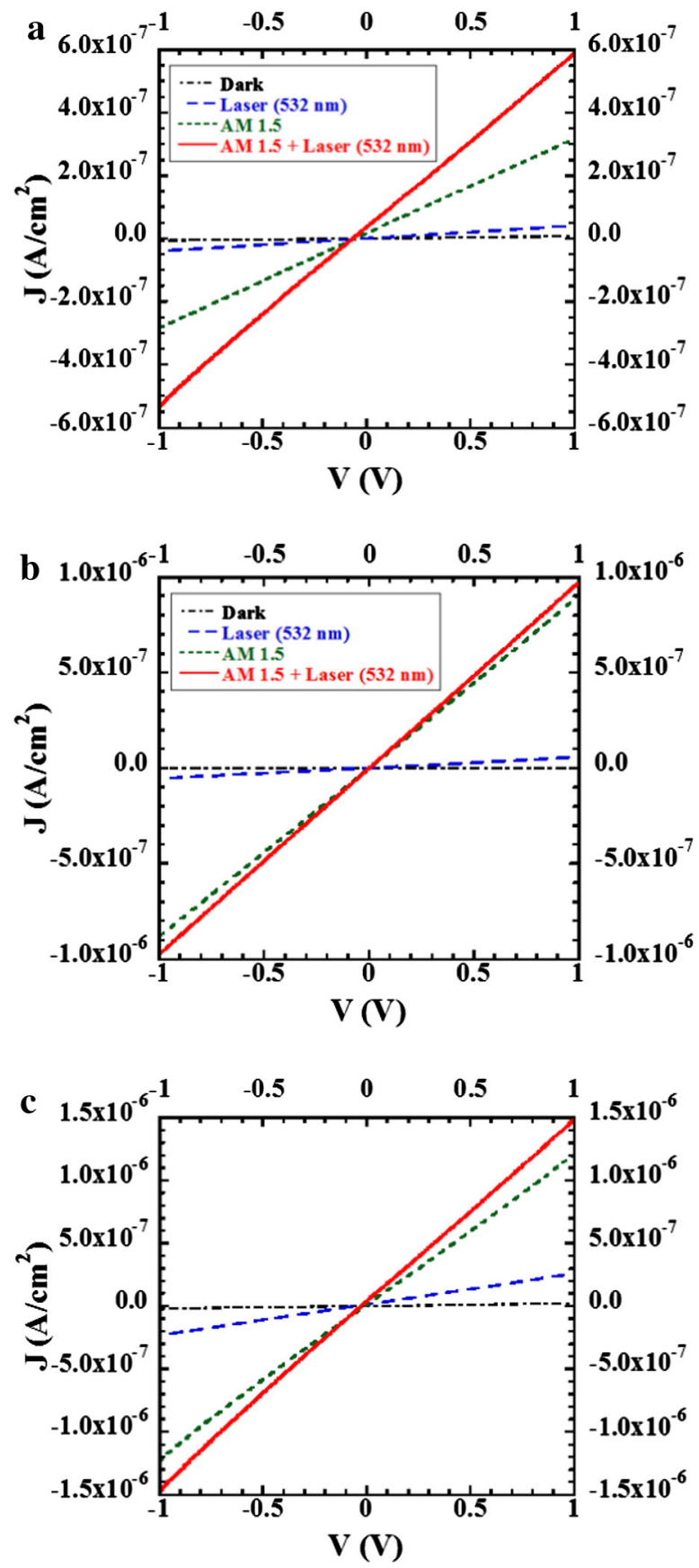

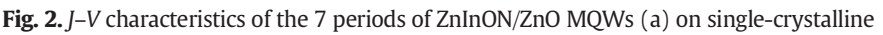
$\mathrm{ZnO}$ template on sapphire substrate and (b) on poly-crystalline $\mathrm{ZnO}$ film on quartz glass substrate and (c) the 15 periods of $\mathrm{ZnInON/ZnO} \mathrm{MQWs} \mathrm{on} \mathrm{single-crystalline} \mathrm{ZnO} \mathrm{template}$ on sapphire substrate.

\subsection{XRD analysis}

To clarify the mechanism in the photo response of MQWs on singlecrystalline $\mathrm{ZnO}$, we performed XRD analysis of the films. Fig. 3(a) shows the $2 \theta-\omega$ scan of symmetric (0002) plane reflection of the ZnInON film on single-crystalline $\mathrm{ZnO}$. The diffraction peaks observed at $2 \theta$ values of $33.7^{\circ}$ and $34.4^{\circ}$ are assigned to the $\mathrm{ZnInON}$ (0002) plane and $\mathrm{ZnO}$ (0002) plane, respectively, and no other peaks are observed. The ZnInON film on single-crystalline $\mathrm{ZnO}$ is highly oriented along c-axis. The tilt angle in the ZnInON film was evaluated from the rocking curve. Fig. 3(b) shows the rocking curve of symmetric (0002) plane reflection of the ZnInON film. The full width at half maximum is $0.09^{\circ}$, indicating that the $\mathrm{ZnInON}$ film has a high crystallinity with good out-of-plane alignment.
We also examined the in-plane epitaxial relationship between the ZnInON film and the single-crystalline ZnO. Fig. 3(c) shows the $\varphi$ scan of the asymmetric (10-11) plane reflection of the ZnInON film. The ZnInON film has a six-fold symmetry without $30^{\circ}$-rotated domains, indicating that the $\mathrm{ZnInON}$ film grows epitaxially on single-crystalline ZnO. Fig. 3(d) shows a reciprocal space map around (10-15) plane of $\mathrm{ZnInON}$ film on single-crystalline $\mathrm{ZnO}$. The $\mathrm{ZnInON}$ film has the same m-lattice parameter of $0.563 \mathrm{~nm}$ as $\mathrm{ZnO}$ and a c-lattice parameter of $0.532 \mathrm{~nm}$. The c-lattice parameter increases from $0.522 \mathrm{~nm}$ given from Vegard's law to $0.532 \mathrm{~nm}$, which indicates the coherent growth of the $\mathrm{ZnInON}$ film on single-crystalline $\mathrm{ZnO}$. The coherent growth is crystal growth, which permits lattice continuity between films and latticemismatched substrates. Crystal strain owing to the coherent growth of films on lattice-mismatched substrates induces the piezoelectric field in the films. Therefore, a strong piezoelectric field is expected to exist in the coherent $\mathrm{ZnInON}$ film due to a large piezoelectric constant of ZnInON.

Such piezoelectric field is also expected to exist in $\mathrm{ZnInON} / \mathrm{ZnO}$ MQWs on single-crystalline $\mathrm{ZnO}$ in Section 3.1. The piezoelectric field is deduced to be a few tens of $\mathrm{MV} / \mathrm{cm}^{2}$ from the piezoelectric constants of $\mathrm{ZnO}$ and $\mathrm{InN}$. The piezoelectric field in the well layers reduces the electron-hole wavefunction overlap, which leads to reduction of the carrier recombination rate and prolongs carrier lifetime in the well layers [33]. The green laser light can excite the carriers in the well layers to the barrier layers, when the carrier lifetime is long enough. Since the piezoelectric field prolongs carrier lifetime in the well layers, the conductivity of 7-periods of MQWs on single-crystalline $\mathrm{ZnO}$ is enhanced by superimposing the green laser light as described in Section 3.1. The enhancement becomes small for 15 periods of MQWs on single-crystalline $\mathrm{ZnO}$ because $\mathrm{ZnInON}$ is partially relaxed by increasing the number of MQWs, and hence the piezoelectric field becomes small. The ZnInON film deposited on the poly-crystalline $\mathrm{ZnO}$ is fully lattice-relaxed (not shown here). Superposition of the green laser light has little effects on the conductivity of MQWs on polycrystalline $\mathrm{ZnO}$.

\subsection{Current-voltage characteristics of MQW solar cells with ZnInON}

We measured the current-voltage characteristics of solar cells with ZnInON/ZnO MQWs in the intrinsic layer of p-i-n structure. Figs. 4(a) and (b) show the characteristics of MQW solar cells on the ptype GaN template and on the p-type Si substrate. The photovoltaic behavior of solar cells with $\mathrm{ZnInON} / \mathrm{ZnO} \mathrm{MQWs}$ is observed, indicating that the MQWs functions as an intrinsic layer of p-i-n solar cell structure. Under solar simulator light, Voc and Jsc of the solar cell on p-Si are $0.23 \mathrm{~V}$ and $7.1 \times 10^{-4} \mathrm{~A} / \mathrm{cm}^{2}$, respectively. Its fill factor (FF) and conversion efficiency are 0.23 and $3.7 \times 10^{-2} \%$. The characteristics under solar simulator light together with green laser light are almost the same as those under solar simulator light. The Voc and the Jsc of the solar cell on p-GaN are increased from $0.16 \mathrm{~V}$ to $0.19 \mathrm{~V}$ and from $1.9 \times 10^{-6} \mathrm{~A} / \mathrm{cm}^{2}$ to $2.5 \times 10^{-6} \mathrm{~A} / \mathrm{cm}^{2}$ by superimposing the green laser light to the solar simulator light. The FF and the conversion efficiency are enhanced from 0.28 to 0.29 and from $8.8 \times 10^{-5 \%}$ to $1.3 \times 10^{-4} \%$ by the superimposition. Fig. 5(a) shows $2 \theta-\omega$ scan of symmetric (0002) plane reflection of the ZnInON films deposited directly on p-GaN and p-Si. The c-lattice parameter of the ZnInON on $\mathrm{GaN}$ is extended compared with that of the ZnInON film on p-Si, which shows that the ZnInON film on GaN is strained. Fig. 5(b) shows the $\varphi$ scan of the asymmetric (10-11) plane reflection of the ZnInON films on p-GaN and p-Si, indicating the epitaxial growth of the ZnInON film on GaN as mentioned above. Therefore, a piezoelectric field is generated in the ZnInON film on GaN. On the other hand, no epitaxial relationship between $\mathrm{ZnInON}$ and $\mathrm{Si}$ is observed. This may be because of the formation of amorphous SiOx layers at ZnInON/Si interfaces that interrupt the epitaxial continuity of ZnInON and Si. In such cases, the lattice parameter of ZnInON films is not influenced by Si substrates, 

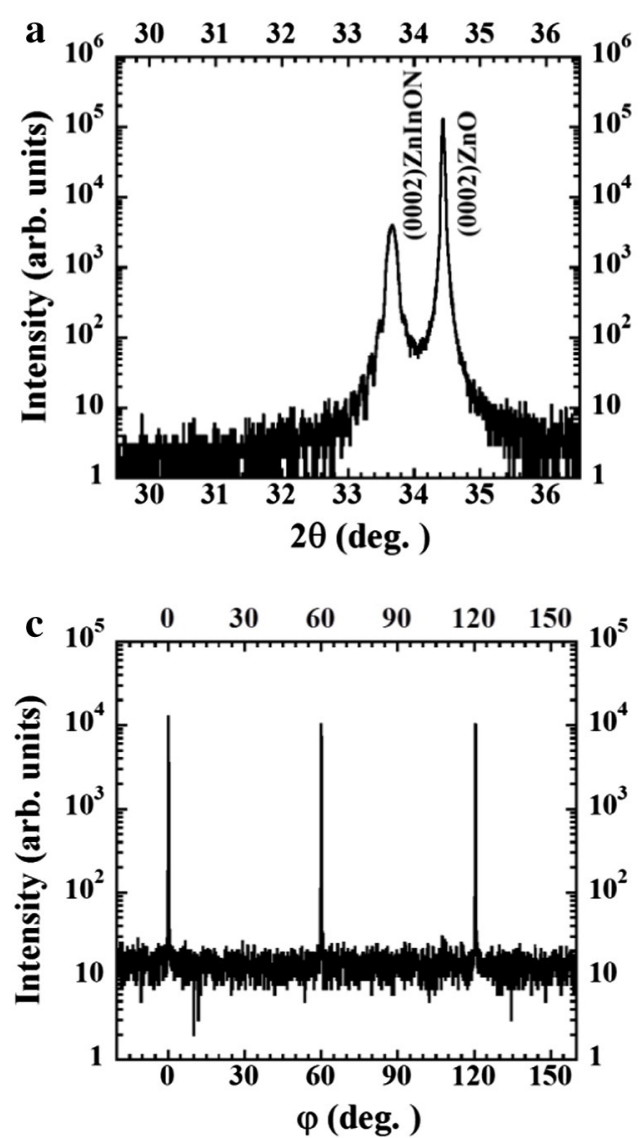

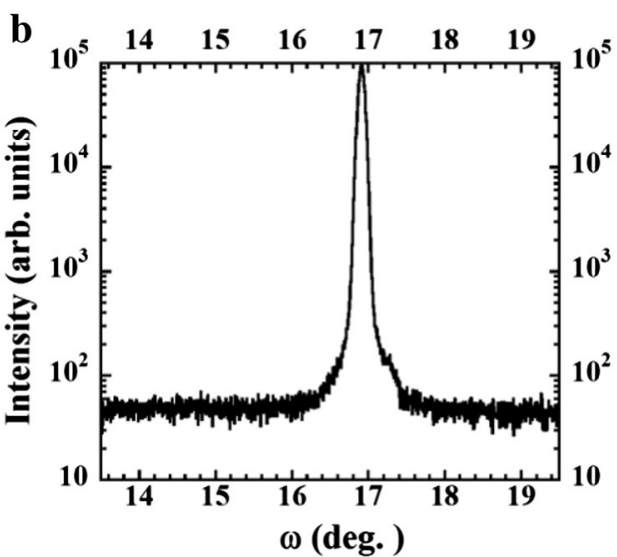

d

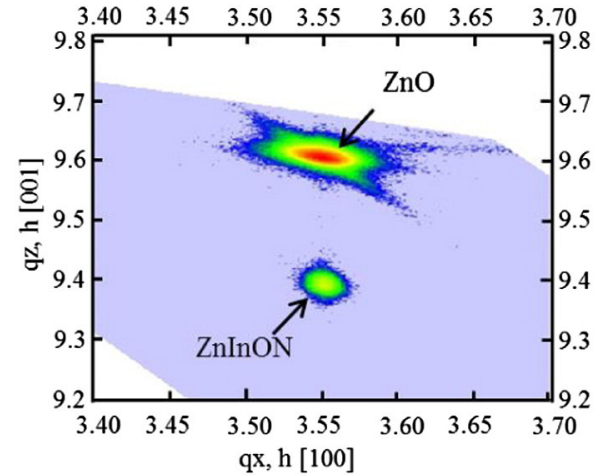

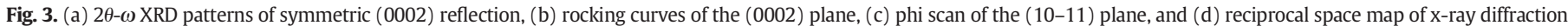
intensities around (10-15) plane of ZnInON film on single-crystalline ZnO template on sapphire substrate.

and no strain is imposed by the lattice mismatch. Therefore, piezoelectric field is not generated in $\mathrm{ZnInON}$ films fabricated on Si. As discussed in Sections 3.1 and 3.2, carrier lifetime is extended by the piezoelectric field in the MQWs, and hence carrier extraction is increased by superimposing the green laser light.

The piezoelectric field in the wells is deduced to be $0.89 \mathrm{MV} / \mathrm{cm}$ from the piezoelectric constants of $\mathrm{ZnO}$ and InN. We carried out a carrier lifetime simulation by a simulator SiLENSe using this piezoelectric field. The simulation shows that the carrier recombination rate in the wells with the piezoelectric field is a low rate of $10^{8} \mathrm{~cm}^{-3} \mathrm{~s}^{-1}$ and the corresponding carrier lifetime is $2.5 \times 10^{-8} \mathrm{~s}$, which is 80 times longer than that without the piezoelectric field. The Voc and the Jsc of the solar cell on p-GaN are enhanced under simultaneous irradiation of solar simulator light together with green laser light because photo-carriers generated by solar simulator light in the wells are excited above the barrier potential by the laser light [34-37]. The carrier lifetime in the wells without the piezoelectric field is $3.1 \times 10^{-10} \mathrm{~s}$ due to the fast carrier recombination rate. This recombination rate is much faster than the carrier extraction rate by the green laser light, and hence the laser light has little effects on the Voc and the Jsc of the solar cell on p-Si.

Regarding the MQW solar cells on Si, the high Jsc owes to the small band gap of $1.2 \mathrm{eV}$ (the band gap of $\mathrm{GaN}$ is $3.4 \mathrm{eV}$ ), which allows efficient absorption of solar light. One possible reason for the difference in the Voc between $\mathrm{Si}$ and $\mathrm{GaN}$ is the difference in the dislocation density. The low Voc observed for the solar cells on p-GaN templates may be attributed to the large number of dislocations $\left(10^{9} \mathrm{~cm}^{-2}\right)$ that induce current leakage paths as well as carrier recombination centers, and thus reduce the Voc [38].

In order to realize potentially high Voc and high Jsc of MQW solar cells, we cope with a fabrication of MQWs utilizing coherent ZnInON with a narrow band gap on alternative p-type semiconductors to commercial p-type GaN templates. Additionally, ZnInON/ZnO multiquantum well solar cells with near ideal band gaps for maximum solar energy conversion efficiency must incorporate $\mathrm{ZnInON}$ layers with higher In contents or lower energy band gaps.

\section{Conclusion}

Solar cells with ZnInON/ZnO MQWs in the intrinsic layer of p-i-n structure were fabricated by RF magnetron sputtering. The MQW solar cells have been deposited on two types of templates; one is p-type GaN template, and the other is p-type Si substrate. The Voc and the Jsc of the MQW solar cell on the p-type Si substrate are $0.23 \mathrm{~V}$ and $7.1 \times 10^{-4} \mathrm{~A} / \mathrm{cm}^{2}$ by superimposing the green laser light to the solar simulator light. While, the Voc and the Jsc of the MQW solar cell on the p-type GaN template are increased from $0.16 \mathrm{~V}$ to $0.19 \mathrm{~V}$ and from $1.9 \times 10^{-6} \mathrm{~A} / \mathrm{cm}^{2}$ to $2.5 \times 10^{-6} \mathrm{~A} / \mathrm{cm}^{2}$ by the superimposition. The piezoelectric field in the coherently grown $\mathrm{ZnInON}$ well layers reduces the electron-hole wavefunction overlap, which decreases the recombination rate. These results show that the long carrier lifetime in the strained ZnInON well layers on p-type GaN template contributes to the enhancement in the photovoltaic effects by the superimposition.

\section{Acknowledgments}

We wish to thank Messrs. K. Utsumi, K. Omi, and T. Shibutami for skillful technical assistance and fruitful discussion. This work was partially supported by Japan Society for the Promotion of Science (JSPS) Grant-in-Aid for Scientific Research, Japan Science and Technology Agency (JST) PRESTO, Grant-in-Aid for JSPS Fellows, and Kyushu University Interdisciplinary Programs in Education and Projects in Research Development. 

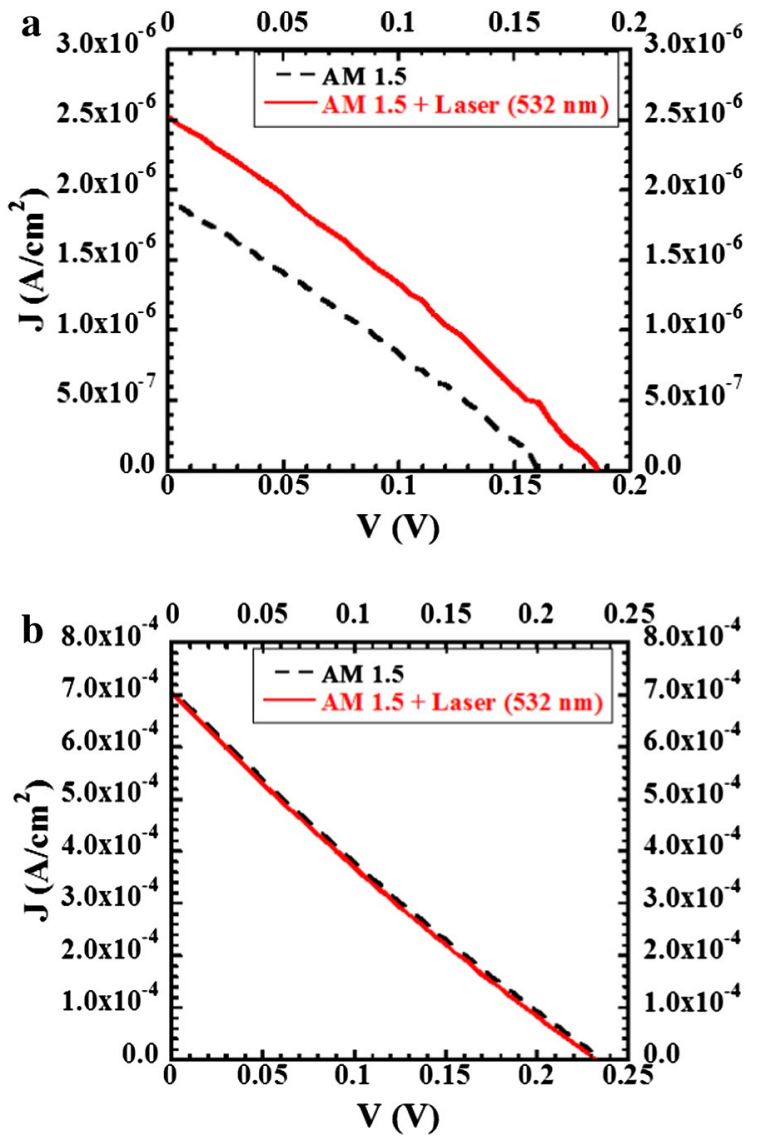

Fig. 4. J-V characteristics of solar cells with ZnInON/ZnO MQW (a) on p-type GaN template on sapphire substrate and (b) on p-type Si substrate.

\section{References}

[1] M.A. Green, Third generation photovoltaics: ultra-high conversion efficiency at low cost, Prog. Photovolt. Res. Appl. 9 (2001) 123.

[2] M.A. Green, Third generation photovoltaics: solar cells for 2020 and beyond, Phys. E. 14 (2002) 65.

[3] G. Conibeer, M. Green, R. Corkish, Y. Cho, E.C. Cho, C.W. Jiang, T. Fangsuwannarak, E. Pink, Y. Huang, T. Puzzer, T. Trupke, B. Richards, A. Shalav, K.L. Lin, Silicon nanostructures for third generation photovoltaic solar cells, Thin Solid Films 511-512 (2006) 654

[4] G. Uchida, K. Yamamoto, M. Sato, Y. Kawashima, K. Nakahara, K. Kamataki, N. Itagaki, K. Koga, M. Shiratani, Effect of nitridation of Si nanoparticles on the performance of quantum-dot sensitized solar cells, Jpn. J. Appl. Phys. 51 (2012) $01 A D 01$.

[5] H. Seo, Y. Wang, M. Sato, G. Uchida, K. Kamataki, N. Itagaki, K. Koga, M. Shiratani, Improvement of Si adhesion and reduction of electron recombination for Si quantum dot-sensitized solar cells, Jpn. J. Appl. Phys. 52 (2013) 01AD05.

[6] W. Shockley, H.J. Queisser, Detailed balance limit of efficiency of p-n junction solar cells, J. Appl. Phys. 32 (1961) 510.

[7] M.A. Green, K. Emery, Y. Hishikawa, W. Warta, E.D. Dunlop, Solar cell efficiency tables (version 39), Prog. Photovolt. 20 (2012) 12.

[8] R.R. King, D.C. Law, K.M. Edmondson, C.M. Fetzer, G.S. Kinsey, H. Yoon, R.A. Sherif, N.H. Karam, 40\% efficient metamorphic GaInP/GalnAs/Ge multijunction solar cells, Appl. Phys. Lett. 90 (2007) 183516.

[9] K. Barnham, I. Ballard, J. Barnes, J. Connolly, P. Griffin, B. Kluftinger, J. Nelson, E. Tsui, A. Zachariou, Quantum well solar cells, Appl. Surf. Sci. 113-114 (1997) 722.

[10] L. Cuadra, A. Marti, A. Luque, Present status of intermediate band solar cell research, Thin Solid Films 451-452 (2004) 593.

[11] T. Kirchartz, K. Seino, J.M. Wagner, U. Rau, F. Bechstedt, Efficiency limits of Si/SiO2 quantum well solar cells from first-principles calculations, J. Appl. Phys. 105 (2009) 104511.

[12] N.G. Anderson, On quantum well solar cell efficiencies, Phys. E. 14 (2002) 126.

[13] K.W.J. Barnham, G. Duggan, A new approach to high-efficiency multi-band-gap solar cells, J. Appl. Phys. 67 (1990) 3490.

[14] J.C. Rimada, L. Hernández, J.P. Connolly, K.W.J. Barnham, Conversion efficiency enhancement of AlGaAs quantum well solar cells, Microelectron. J. 38 (2007) 513.

[15] H. Fujii, K. Watanabe, M. Sugiyama, Y. Nakano, Effect of quantum well on the efficiency of carrier collection in InGaAs/GaAsP multiple quantum well solar cells, Jpn. J. Appl. Phys. 51 (2012) 10ND04.

[16] R. Dahal, J. Li, K. Aryal, J.Y. Lin, H.X. Jiang, InGaN/GaN multiple quantum well concentrator solar cells, Appl. Phys. Lett. 97 (2010) 073115.
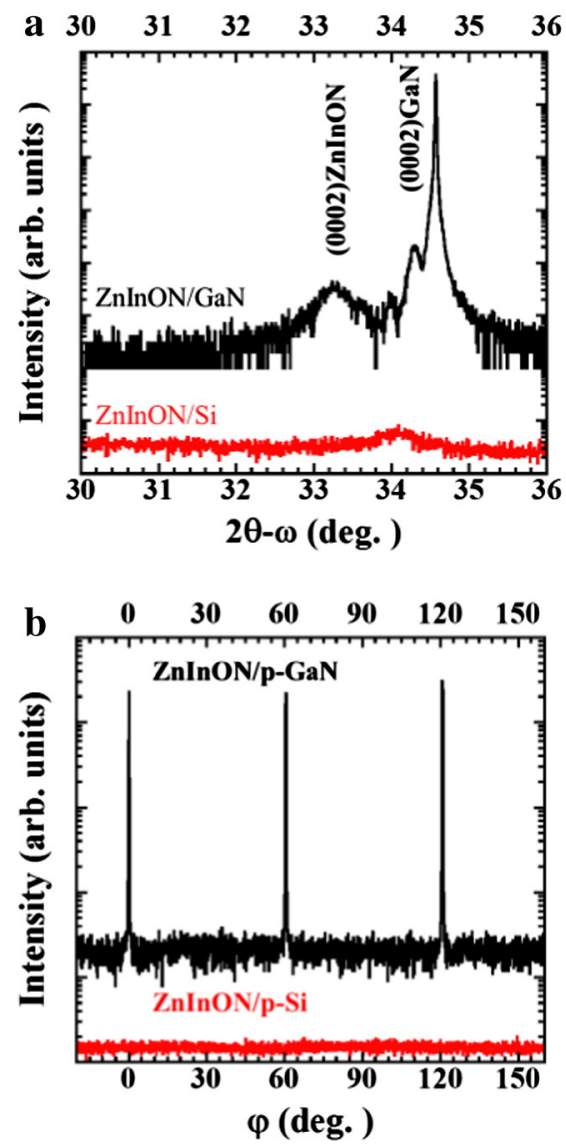

Fig. 5. (a) 2 $2 \theta-\omega$ XRD patterns of symmetric (0002) reflection and (b) phi scan of the (10-11) plane of ZnInON film on p-GaN template and p-Si substrate.

[17] R.M. Farrell, C.J. Neufeld, S.C. Cruz, J.R. Lang, M. Iza, S. Keller, S. Nakamura, S.P. DenBaars, U.K. Mishra, J.S. Speck, High quantum efficiency InGaN/GaN multiple quantum well solar cells with spectral response extending out to $520 \mathrm{~nm}$, Appl. Phys. Lett. 98 (2011) 201107.

[18] A. Luque, A. Marti, L. Cuadra, Thermodynamic consistency of sub-bandgap absorbing solar cell proposals, IEEE Trans. Electron Devices 48 (2001) 2118.

[19] N. Itagaki, T. Iwasaki, M. Watanabe, T. Den, "Metal oxynitride semiconductor containing zinc", U.S. Patent No. 8274078 (2008).

[20] N. Itagaki, M. Shiratani, G. Uchida, "Multi-quantum well solar cell and method of manufacturing multi-quantum well solar cell”, PCT/JP2013/055973, 2013.

[21] K. Matsushima, T. Hirose, K. Kuwahara, D. Yamashita, G. Uchida, H. Seo, K. Kamataki, K. Koga, M. Shiratani, N. Itagaki, Epitaxial growth of ZnInON films with tunable band gap from 1.7 to $3.3 \mathrm{eV}$ on ZnO templates, Jpn. J. Appl. Phys. 52 (2013) 11NM06

[22] A.G. Bhuiyan, A. Hashimoto, A. Yamamoto, Indium nitride $(\operatorname{InN})$ : a review on growth, characterization, and properties, J. Appl. Phys. 94 (2003) 2779.

[23] N. Itagaki, K. Kuwahara, K. Nakahara, D. Yamashita, G. Uchida, K. Koga, M. Shiratani, Highly conducting and very thin $\mathrm{ZnO}$ :Al films with $\mathrm{ZnO}$ buffer layer fabricated by solid phase crystallization from amorphous phase, Appl. Phys. Express 4 (2011) 011101.

[24] K. Kuwahara, N. Itagaki, K. Nakahara, D. Yamashita, G. Uchida, K. Kamataki, K. Koga, M. Shiratani, High quality epitaxial ZnO films grown on solid-phase crystallized buffer layers, Thin Solid Films 520 (2012) 4674.

[25] D.C. Look, K.D. Leedy, A. Kiefer, B. Claflin, N. Itagaki, K. Matsushima, I. Surhariadi, Model for thickness dependence of mobility and concentration in highly conductive zinc oxide, Opt. Eng. 52 (2013) 033801.

[26] N. Itagaki, K. Kuwahara, K. Matsushima, K. Oshikawa, Novel fabrication method for ZnO films via nitrogen-mediated crystallization, Proc. SPIE 8263 (2012) 826306.

[27] N. Itagaki, K. Kuwahara, Novel fabrication method of $\mathrm{ZnO}$ films utilizing solid-phase crystallized seed layers, MRS Proc. 1315 (2011) 15.

[28] I. Suhariadi, K. Oshikawa, K. Kuwahara, K. Matsushima, D. Yamashita, G. Uchida, K. Koga, M. Shiratani, N. Itagaki, Effects of Nitrogen on Crystal Growth of SputterDeposited ZnO Films for Transparent Conducting Oxide, Jpn. J. Appl. Phys. 52 (2013) $11 \mathrm{NB} 03$.

[29] S. Bornholdt, N. Itagaki, K. Kuwahara, H. Wulff, M. Shiratani, H. Kersten, Characterization of the energy flux toward the substrate during magnetron sputter deposition of ZnO thin films, Plasma Sources Sci. Technol. 22 (2013) 025019.

[30] I. Suhariadi, K. Matsushima, K. Kuwahara, K. Oshikawa, D. Yamashita, H. Seo, G. Uchida, K. Kamtaki, K. Koga, M. Shiratani, S. Bornholdt, H. Kersten, Harm Wulff, N. 
Itagaki, Effects of Hydrogen Dilution on ZnO Thin Films Fabricated via NitrogenMediated Crystallization, Jpn. J. Appl. Phys. 52 (2013) 01 AC08.

[31] I. Suhariadi, N. Itagaki, K. Kuwahara, K. Oshikawa, D. Yamashita, G. Uchida, K. Kamataki, K. Koga, K. Nakahara, M. Shiratani, ZnO:Al thin films with buffer layers fabricated via nitrogen mediated crystallization: effects of N2/Ar gas flow rate ratio, Trans. Mater. Res. Soc. Jpn. 37 (2012) 165.

[32] S.B. Zhang, S.H. Wei, A. Zunger, Intrinsic n-type versus p-type doping asymmetry and the defect physics of ZnO, Phys. Rev. B 63 (2001) 075205.

[33] N. Tansu, H. Zhao, G. Liu, X.H. Li, J. Zhang, H. Tong, Y.K. Ee, III-Nitride photonics, IEEE Photonics J. 2 (2010) 2045887.

[34] T. Kuroda, A. Tackeuchi, Influence of free carrier screening on the luminescence energy shift and carrier lifetime of InGaN quantum wells, J. Appl. Phys. 92 (2002) 3071.

[35] A. Martí, E. Antolín, C.R. Stanley, C.D. Farmer, N. López, P. Díaz, E. Cánovas, P.G. Linares, A. Luque, Production of photocurrent due to intermediate-to-conduction- band transitions: a demonstration of a key operating principle of the intermediate-band solar cell, Appl. Rev. Lett. 97 (2006) 247701.

[36] K. Ueno, S. Juodkazis, T. Shibuya, Y. Yokot, V. Mizeikis, K. Sasaki, H. Misawa, Nanoparticle plasmon-assisted two-photon polymerization induced by incoherent excitation source, J. Am. Chem. Soc. 130 (22) (2008) 6928.

[37] N. Ahsan, N. Miyashita, M.M. Islam, K.M. Yu, W. Walukiewicz, Y. Okada, Two-photon excitation in an intermediate band solar cell structure, Appl. Phys. Lett. 100 (2012) 172111.

[38] R.H. Horng, S.T. Lin, Y.L. Tsai, M.T. Chu, W.Y. Liao, M.H. Wu, R.M. Lin, Y.C. Lu, Improved conversion efficiency of GaN/InGaN thin-film solar cells, IEEE Electron Device Lett. 30 (2009) 724. 